\title{
El rigor metodológico en la evaluación curricular
}

\section{Methodological Rigor in the Curricular Evaluation}

\author{
Andrea Ramírez, González. \\ División de Educación Rural \\ Centro de Investigación y Docencia en Educación \\ Universidad Nacional \\ Heredia, Costa Rica \\ aramgo3176@gmail.com
}

Recibido 19 de mayo de $2011 \bullet$ Aceptado 26 de agosto de 2011

\begin{abstract}
Resumen. En el presente ensayo se desarrolla la importancia del rigor metodológico en una evaluación curricular. Se parte de que para su valoración, por parte de los interesados, es exigido que el evaluador muestre el rigor con el que se ha realizado el proceso, de tal manera que sea posible evidenciar elementos que la hacen válida, confiable y creíble. Para ello, se sustenta el rigor de la evaluación con base en la articulación de aspectos significativos, valorativos, teóricos, metodológicos, de participación de los actores y de ética. Estos permiten delimitar y desarrollar el objeto por evaluar. Por lo tanto, cada uno de estos aspectos forman parte esencial del camino a seguir en un proceso evaluativo.
\end{abstract}

Palabras claves. Rigor metodológico, objeto por evaluar, rol y ética del evaluador.

Abstract. This essay develops the importance of the methodological rigor in a curricular evaluation. The assessment of the curricular evaluation by the interested parties has to show the assessor's rigor with which the process has been realized, in this way it is possible to demonstrate elements that make the evaluation valid, reliable and creditable. The rigor of the evaluation is sustained with the articulation of aspects that are significant, assessable, theoretical, and methodological, of participation of the actors and of ethics which allow delimiting and developing the object to be evaluated. Therefore, each of these aspects is an essential part of the way to continue in an evaluative process.

Keywords. Methodological rigor, object to be evaluated, role and ethics of the evaluator.

Licenciada en Enseñanza de la Matemática, Universidad de Costa Rica. Máster en Evaluación Educativa de la Universidad de Costa Rica. Académica de la División de Educación Rural de la Universidad Nacional de Costa Rica. Investigadora de temáticas relacionadas con el perfil de docentes de secundaria y primara, formación de docentes para el ámbito rural. Dirección postal: San José, Cantón de Mora, Costa Rica, 200 metros este y 25 sur Servicentro Colón, Ciudad Colón. Número de teléfono o celular: (506) 22490472, (506) 88112473. 


\begin{abstract}
A los investigadores de evaluación cuantitativa les gusta acusar de falta de precisión a los investigadores de evaluación cualitativa. La acusación no distingue entre precisión y una exactitud más amplia. Las respuestas pueden ser precisas pero falsas.
\end{abstract}

Ian F. Shaw

\section{El "rigor metodológico"}

A partir de los aportes de Alicia de Alba en el libro Evaluación curricular: conformación conceptual del campo, se trata el tema de la investigación y la evaluación. Se plantea la valoración de una situación o proceso social como la tarea fundamental de la evaluación, la cual, necesariamente, se vincula a la actividad investigativa, por cuanto:

(...) realizar un análisis conceptual del objeto a evaluar y al construir una síntesis, tanto conceptual como teórica, sobre el mismo, se está produciendo conocimiento sobre el objeto evaluado. (De Alba, 1991, p. 93)

En este sentido, De Alba considera la evaluación como investigación evaluativa, porque conjuga aspectos relacionados con la producción de conocimiento, la valoración y el compromiso.

De acuerdo con Weiss, citada por Picado (2006, p. 6), la distancia entre evaluación e investigación es muy corta, pues ambas comparten la rigurosidad en el uso de métodos y técnicas para recolectar y analizar la información.

En consecuencia, la evaluación, asumida como investigación evaluativa, no escapa a una preocupación latente en los investigadores evaluativos, cual es mostrar la rigurosidad científica que respalda los hallazgos obtenidos. Por ello, de todo el cuerpo de la evaluación, toma especial importancia juzgar el proceso metodológico seguido, de tal manera que se valoren cada uno de los aspectos desarrollados en relación con las conclusiones planteadas.

En este proceso de juzgamiento de lo metodológico, se considera como elemento esencial el rigor metodológico con que fue desarrollada la evaluación.

Para evaluar el rigor metodológico, se presuponen criterios de rigurosidad científica que deben ser “(...) coherentes con los propósitos, fines y bases filosóficas del paradigma [que representan]" Gurdián (2007, p. 244).

Así, desde el paradigma cuantitativo, los criterios de validez y confiabilidad permiten evidenciar una evaluación rigurosa.

Desde el paradigma cualitativo, autores como Guba y Lincoln (1981), citados por Gurdián (2007), formulan cuatro criterios que permiten evaluar el rigor científico de una investigación mediante la analogía con aquellos criterios establecidos del paradigma cuantitativo. Estos criterios son credibilidad, transferibilidad, dependebilidad, confirmabilidad.

La credibilidad se logra cuando es posible confirmar que los hallazgos son verdaderos para los participantes y los relacionados con el estudio. Se asocia con la validez interna en la que se consideran las verdades de la investigación. 
La transferibilidad es la posibilidad de ajustar los resultados a otros contextos similares al realizado en la investigación, es decir, es la validez externa de la investigación, por cuanto muestra la aplicabilidad y generalización de los resultados.

La dependebilidad es el control externo del proceso de la investigación.

La confirmabilidad se refiere a que se examinen los datos obtenidos por otro u otra investigadora con posicionamiento similar de lo investigado y se logren las mismas conclusiones. Esto conduce a la objetividad que debe reflejarse desde el accionar de quien investiga.

El cumplimiento de cada uno de los criterios anteriores permite mostrar que el proceso y los resultados de una investigación tienen asidero científico y, por lo tanto, la información que se produjo es creíble.

Por otra parte, la rigurosidad metodológica no se ajusta únicamente a lo metodológico, es decir, al camino recorrido en la evaluación, pues la rigurosidad se expande a otros aspectos del cuerpo de la evaluación -entendida como investigación evaluativa-, pues se ha asumido en este escrito la posición de De Alba (1991) sobre la conjugación de aspectos teóricos y aspectos axiológicos como inherentes y esenciales en la evaluación. Desde esta perspectiva, la opción metodológica escogida forma parte de la culminación de la delimitación del objeto por evaluar.

Asimismo, De Alba conduce el carácter teórico y el carácter axiológico de la evaluación al campo de la evaluación curricular, por lo que las ideas siguientes se circunscriben a este campo.

\section{Delimitación del objeto por evaluar}

Los aportes de De Alba (1991) en torno a la evaluación curricular, concebida como corte y articulación de los discursos del currículum y la evaluación en el contexto de las ciencias humanas, parten de la noción de totalidad desde una perspectiva epistemológica. Esta perspectiva refiere a una posición constante frente a la realidad social cambiante y dinámica que posibilita delimitar un campo de problematización y observación, desde una construcción teórica articulada del problema a evaluar.

La delimitación de los campos de observación y problematización permiten definir el objeto a evaluar, porque perfilan un preliminar abordaje teórico-conceptual a partir del cual se construye el objeto, y del cual se infiere la definición metodológica y técnica asumida. Estos campos deben ser coherentes con las características teórico-axiológicas del objeto a evaluar, pues son los elementos constitutivos de la evaluación, por cuanto la interrelación de lo axiológico y lo teórico permite conformar "el juicio que se realiza en la evaluación".

De Alba define el carácter teórico de la evaluación como una "comprensión conceptual desde una posición teórica que permite la comprensión de lo evaluado" (1991, p. 95). Para esta autora, la importancia del sustento teórico en la evaluación radica en que se disminuye la posibilidad de caer en procesos de enjuiciamiento y no de evaluación.

El carácter axiológico de la evaluación lo refiere al "conjunto de valores a partir de los cuales se analiza el objeto-sujeto a evaluar" (De Alba, 1991, p. 98). En este conjunto, se relacionan aspectos de la dimensión social, política, ideológica, cultural, económica, así como del rol, responsabilidad y compromiso que ocupan los evaluadores y las evaluadoras en el proceso. 
Es importante considerar un balance entre lo teórico y lo axiológico, pues la construcción de los juicios se determina sobre la base de la comprensión teórica y axiológica de la evaluación realizada.

Otro elemento fundamental en la aprehensión inicial de una evaluación curricular es el contexto y la determinación de aspectos relevantes en que se realiza esta; es decir, lo que De Alba denomina significatividad.

Partir de lo significativo de una evaluación permite “(...) la apertura-aprehensión inicial y la delimitación-construcción del objeto" (De Alba, 1991, p. 135).

Esta aprehensión de la realidad del objeto por evaluar invita a cuestionarse, en un primer momento, el porqué y el para qué de la evaluación, y sobre quiénes la pueden realizar. Esto permite concretar aún más la problemática que se pretende evaluar y es un medio para acercarse a la noción de significatividad del proceso.

Cabe resaltar, que es necesario analizar la significatividad desde el rigor de la posición teórica y axiológica asumida, con la finalidad de que se comprenda y se valore con mayor precisión e importancia en el contexto de la evaluación.

En este proceso de delimitación del objeto a evaluar, De Alba (1991) propone que se realice un análisis global de la lectura de la realidad en la que se consideran dimensiones generales y específicas; planos estructural-formal y procesal-práctico; niveles de significación explícita e implícita. De esta forma es posible puntualizar los aspectos medulares del análisis curricular.

Las dimensiones generales son la dimensión social amplia, la dimensión institucional, y la dimensión didáctica.

La dimensión social amplia articula aspectos culturales, políticos, ideológicos, sociales, económicos que constituyen el currículo.

La dimensión institucional refiere a la oportunidad que tiene la institución de potenciar sus capacidades para impulsar nuevos proyectos a partir de la evaluación curricular realizada.

La dimensión didáctica representa las dimensiones anteriores reflejadas en la relación del maestro y del estudiante, en la interacción grupal y la apropiación de los contenidos.

El plano desde lo estructural formal se centra en el análisis de planes de estudio, los programas, legislación, reglamentos. El plano desde lo procesal práctico conlleva aspectos relacionados con la práctica curricular.

Los niveles de significación incorporan manifestaciones de la cultura, el lenguaje del currículo por evaluar.

Al tomar una postura epistemológica en la evaluación curricular, atendiendo el carácter teórico-axiológico, es posible seleccionar la opción metodológica que mejor se ajuste a las características del objeto evaluado. Esta puede sustentarse en el enfoque cuantitativo o cualitativo de la evaluación.

Desde la perspectiva de totalidad asumida por de Alba, se confiere especial interés a la hermenéutica-dialéctica como opción metodológica del enfoque cualitativo que "centra la atención en la comprensión (verstehen) más que en la explicación causal (erklären)" (De Alba, 1991, p. 151).

Esta metodología se caracteriza por fundamentarse en un marco teórico desde el inicio de su formulación y a lo largo del proceso. Permite profundizar en la interpretación mediante el análisis de la información que se realiza. Por tanto, se requiere de suficiente pericia y conocimiento para 
decidir en qué determinado momento de la evaluación se ha logrado el nivel de interpretación óptimo en relación con los resultados obtenidos.

El momento decisivo para considerar la interpretación de los resultados "a término" debe ir acompañado con una reflexión constante acerca de los criterios de validez -señalados en párrafos anteriores-, de tal manera que se constate el rigor metodológico en la evaluación.

También, se han señalado algunos aspectos esenciales en la delimitación del objeto por evaluar, a saber: totalidad, significatividad, epistemología, carácter axiológico, carácter teórico, metodología. Todos estos aspectos son puntos de partida en un proceso de evaluación curricular en el que se requiere de la articulación y construcción simultánea, por ello, el camino a seguir en una evaluación curricular no se visualiza de forma lineal, de secuencia de pasos, sino más bien como la interrelación de estos elementos.

Una forma gráfica que representa la articulación es mediante un diagrama de Ven en el que los círculos que se intersecan, implica que todos los aspectos se afectan entre si.

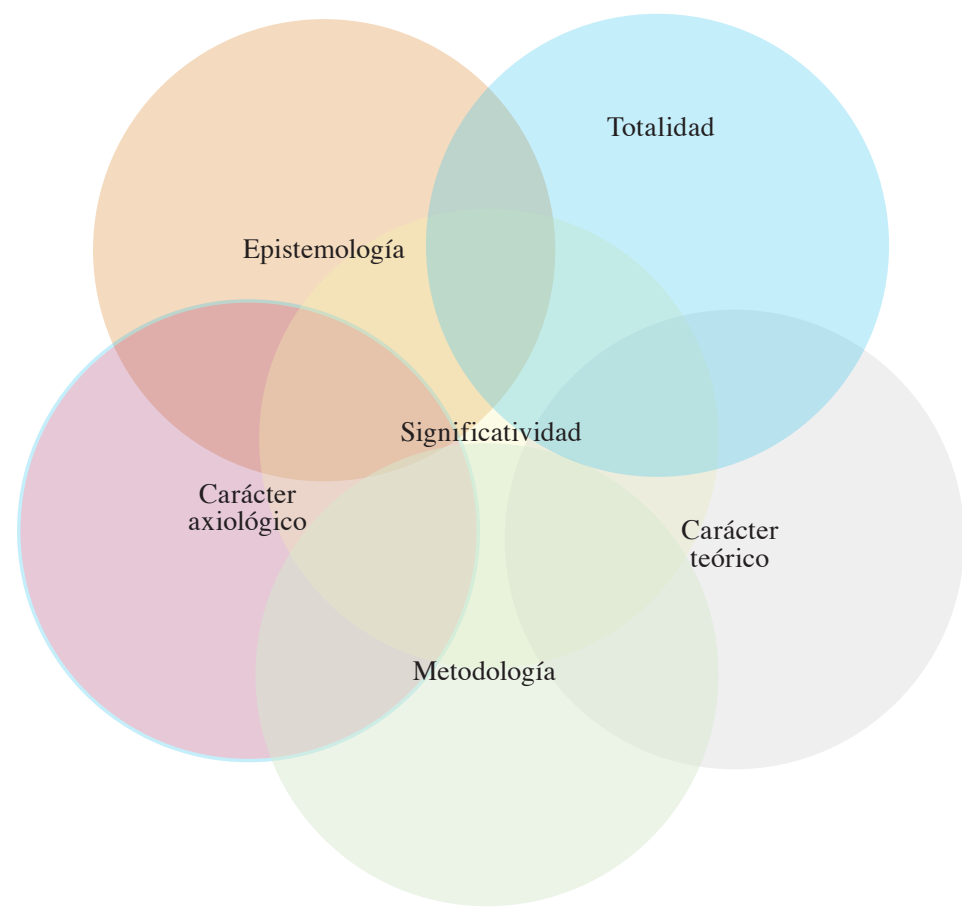

Figura 1: Aspectos en la delimitación del objeto por evaluar.

Nota: Elaboración propia a partir de los referentes aportado por

De Alba (1991) en su concepción de evaluación curricular.

\section{El actuar de la persona que evalúa desde su rol y la ética}

El rol del evaluador o evaluadora en el proceso tiene especial importancia, pues juega un papel fundamental desde su participación en el diseño, la ejecución, conclusión y devolución de resultados de la evaluación. Esto, por cuanto recae sobre esta persona la responsabilidad profesional, ética y moral de conducir, de forma adecuada, una evaluación en la que los hallazgos y recomendaciones 
planteados son el cimiento para la toma de decisiones. Además, debido a que, en múltiples ocasiones, las consecuencias sociales que provoca afectan, de manera sensible, al grupo de interesados o de referencia, que solicitaron y se sometieron a la evaluación.

Así, el actuar del evaluador a lo largo del proceso debe predefinirse y clarificarse, pues permite asumir la posición y responsabilidad que le compete para tal fin.

En concordancia con lo anterior, Carrión (2001) indica cuáles son las tareas que tiene el evaluador o evaluadora en el proceso: a) identificar los valores y características del grupo de referencia en el que se realiza la evaluación; b) crear condiciones técnicas para llevar a cabo la evaluación; c) identificar procedimientos viables en relación con las condiciones políticas y d) seleccionar o construir las técnicas de evaluación más pertinentes.

Carrión señala, además, como tarea fundamental. una acción que fue mencionada al inicio de este ensayo, esto es, "hacer válida la evaluación". En este sentido el evaluador o evaluadora debe generar confianza al grupo de interesados sobre las conclusiones y la veracidad de los juicios a los que se llega, así como el profesionalismo que muestra en relación con el diseño y desarrollo de la evaluación.

Estas tareas se orientan desde el inicio con la posición del profesional ante el corpus axiológico constituido, predefinido y reconstruido que caracteriza a las personas, a las instituciones educativas, a los programas y al evaluador mismo. Por ello, es necesario que los hallazgos o conclusiones de la evaluación sean coherentes con ese sistema de valores. Aún más, las tareas mencionadas se vinculan con aspectos relacionados, en su totalidad, con el carácter teórico y metodológico propuesto por De Alba (1991) y adoptado en este documento.

La función de la persona que evalúa plantea, como principio elemental, el carácter axiológico y valorativo vinculado con la ética de la evaluación.

Algunos principios éticos que, desde la perspectiva de diversos autores, deben ser tomados en cuenta en una evaluación, son citados por Shaw (2003, p. 114):

(...) consentimiento, confidencialidad y derecho a abandonar la investigación en cualquier momento". (Eisner 1991).

(...) relaciona los principios éticos con el respeto mutuo, la no coerción y no manipulación, el respeto y defensa de valores democráticos. (House 1993)

(...) principio ético conservar la libertad humana. Warwick, 1982)

Estos principios de la ética de la evaluación deben considerarse como elementos específicos en el diseño de la evaluación, el trabajo de campo y el análisis (Shaw, 2003, p. 117).

Complementario a los autores anteriores, Carrión (2001) propone algunos cuestionamientos que deben realizarse al pensar éticamente en la evaluación, a saber:

- ¿Qué papel tendrá el evaluador en el conjunto de relaciones políticas y sociales que presenta la [evaluación]?

- ¿Cómo se relacionará con sus interlocutores?

- ¿A quién definirá como interlocutor? (p. 55) 
Estas tres preguntas remiten, de acuerdo con Carrión (2001), a aspectos que se deben considerar acerca de la relación del evaluador o evaluadora con las relaciones políticas y sociales de la institución en la que se realiza la evaluación, la selección, la relación y participación de los informantes de la institución.

Asimismo, se retoma la construcción del sistema axiológico que resguarda la evaluación; la credibilidad de los resultados se apoya en su propio juicio profesional, el cual apela a los principios, valores personales, experiencia, conocimiento de educador y evaluador educativo.

En síntesis, estos argumentos remiten a la ética de la evaluación vinculada con el objeto a evaluar, el proceso y rigor metodológico a seguir y los participantes de la evaluación como elementos constitutivos y articulados en una evaluación curricular.

\section{Reflexión final}

Una evaluación curricular conlleva mucho más que pensar en un diseño de evaluación, pues en ella se conjugan una serie de aspectos epistemológicos, axiológicos, teóricos y metodológicos que sustentan el inicio de una evaluación, pero que también están presentes en el camino que se sigue.

Estos aspectos se articulan sobre la base de un objeto por evaluar que responde a los intereses de algunas personas insertas en una institución, programa o proyecto. Por tanto, la valoración se debe asumir con responsabilidad profesional por parte de la persona que evalúa, porque debe producir y mostrar los hallazgos acerca del objeto evaluado.

El evaluador o evaluadora debe dar cuenta de la transparencia del proceso, de forma que permite hacer válida la evaluación realizada.

\section{Referencias bibliográficas}

Carrión, C. (2001). Valores y principios para evaluar la educación. México: Paidós.

De Alba, A. (1991). Evaluación curricular. Conformación conceptual del campo. México: Universidad Nacional Autónoma de México.

Gurdián, A. (2007). El paradigma cualitativo en la investigación socio-educativa (Colección IDER, CECC, AECI). San José, Costa Rica: PrintCenter.

Picado, M. (2006). Un acercamiento a la evaluación cualitativa. San José, Costa Rica. EUCR.

Shaw, I. (2003). La evaluación cualitativa. Introducción a los métodos cualitativos. Barcelona, España: Paidós. 